\title{
Maximow PY, McDaniel RE, Jordan VC: Tamoxifen. Pioneering medicine in breast cancer
}

\author{
Springer Basel 2013
}

\author{
R. M. Lowenthal
}

Published online: 10 April 2014

(C) Springer Basel 2014

This is a book that had to be written. It tells the story of the development of tamoxifen from a failed 'morning after' pill to an enormously successful drug for the treatment of breast cancer in all its stages. This development proceeded because of the scientific astuteness and dedication of a handful of scientists and physicians who had to battle against the business plans of the original owners of the intellectual property. The story is a mixture of the personal journey of Craig Jordan-the man who is known as the 'father of tamoxifen' and pure science. Although Jordan is listed as the third co-author, this is really his story, which seems to have been partially ghost-written by the other two. This technique leads to some style confusion as the text is composed in a mixture of the first and third persons with the narrative switching from time to time between the two, often unaccountably.

That minor criticism aside, for anyone who has been involved in the treatment of breast cancer over the years, this is a fascinating story. Jordan describes how he fell serendipitously into research on the drug, and how there has been increasing recognition of the value of tamoxifen as a treatment for breast cancer in all its stages (prevention, adjuvant, advanced). The descriptions of research on tamoxifen are equally detailed and up-to-date with respect to both the laboratory and the clinic. Jordan's work could be described as the epitome of so-called translational research, illustrating just how to move a treatment from 'bench to bedside'. Intriguingly, similar to the story of some more recent breakthrough cancer drugs (such as imatinib and filgrastim), the pharmaceutical company involved in tamoxifen's early development had to be cajoled into bringing the drug to market, against their expectations that there was little likelihood of it benefitting their bottom line. Millions of patients later, and no doubt mega-millions of dollars later, tamoxifen has become probably the biggest selling anti-cancer drug of all time. This success is well deserved, as is explained well in the book. Forty years on, the drug is still widely used for treatment of breast cancer and has well and truly stood the test of time.

In retrospect, tamoxifen could be considered the first successful 'targeted treatment' for cancer, although at the time of its initial testing, in the 1970s, the idea of treating cancer by use of a drug acting on a narrow 'target' did not even exist. This statement may surprise the current generation of cancer scientists, for whom the identification of a target and the subsequent logical development of a chemical agent to interfere with that target's function are the sine qua nons of their existence.

This book should be available in every oncology department. The story will interest clinicians and laboratory scientists alike. It has both historical value and value as a guide that will illuminate the path to successful involvement in cancer research for younger cancer researchers.

R. M. Lowenthal $(\bowtie)$

Menzies Research Institute, University of Tasmania, Tasmania, Australia

e-mail: R.M.Lowenthal@utas.edu.au 